\title{
An Evaluation of the Benefit of Cytomegalovirus Prophylaxis with Acyclovir on Post-Transplant Cytomegalovirus Infection Prevention in a Population of Renal Transplant Recipients in Nigeria
}

\author{
Nnamdi Chuks Menakaya \\ Medicine and Occupational Health Clinic, 11 PLC, Lagos, Nigeria \\ Email: ncmenakaya@gmail.com
}

How to cite this paper: Menakaya, N.C. (2021) An Evaluation of the Benefit of Cytomegalovirus Prophylaxis with Acyclovir on Post-Transplant Cytomegalovirus Infection Prevention in a Population of Renal Transplant Recipients in Nigeria. Open Journal of Nephrology, 11, 437-449.

https://doi.org/10.4236/ojneph.2021.113036

Received: August 6, 2021

Accepted: September 26, 2021

Published: September 29, 2021

Copyright $\odot 2021$ by author(s) and Scientific Research Publishing Inc. This work is licensed under the Creative Commons Attribution International License (CC BY 4.0).

http://creativecommons.org/licenses/by/4.0/

\begin{abstract}
Background: Cytomegalovirus (CMV) is an important infection in renal transplant recipients and may significantly impact recipients' long-term outcome and graft survival. Objective: This study aimed to evaluate the benefit of prophylaxis with acyclovir on post-transplant CMV infection prevention in a population of renal transplant recipients in Lagos, Nigeria. Subjects and Methods: The study was a cross-sectional design involving renal transplant recipients attending post-transplant follow-up clinics in Lagos, Nigeria between October 2004 and July 2005. Data on the use of CMV prophylaxis were obtained from the hospital case records of the study subjects. Enzyme-Linked Immunosorbent Assay (ELISA) was employed to detect CMV IgM antibodies for the diagnosis of post-transplant CMV infection and Microsoft Excel and EPI-Info 2002 statistical software were used for data entry and analysis. Results: Forty (40) renal transplant recipients were studied, 32 recipients were males and 8 were females with M:F ratio of 4:1. The mean age of the recipients was $39 \pm 11.6$ years old. The recipients' post-transplant duration ranged from 2 to 80 months (Mean $17.6 \pm 18.6$ months). Fifteen (37.5\%) of the transplant recipients received acyclovir prophylaxis for six months, one recipient $(2.5 \%)$ received ganciclovir prophylaxis for three weeks while 24 recipients $(60 \%)$ received no prophylactic therapy. There was no significant difference in the prevalence of seropositive CMV-IgM between transplant recipients who used CMV prophylaxis and those who did not (Fisher exact $\mathrm{p}=$ 0.45). Conclusion: Prophylaxis with acyclovir for six months showed no significant benefit on post-transplant CMV infection prevention in renal transplant recipients.
\end{abstract}




\section{Keywords}

Post-Transplant Cytomegalovirus Infection, Prophylaxis, Acyclovir, Renal Transplant Recipients, Nigeria

\section{Introduction}

The advent of renal transplantation almost seven decades ago provided the best opportunity for returning to healthy and productive life for patients with end-stage renal disease [1]. Since the first successful renal transplant in Nigeria was carried out at Saint Nicholas Hospital, a private medical facility in Lagos over two decades ago, several other transplant centres from different geographical regions of the country are now actively carrying out kidney transplants with over two hundred renal transplants carried out between them [2].

Cytomegalovirus (CMV) infection is an important infection in renal transplant recipients [3] [4] [5]. Studies have estimated the incidence of CMV infection in the renal transplant population to be between $8 \%$ and $32 \%$ [6] [7]. In the renal transplant population, CMV infection can occur as a de novo infection or as a reactivation of the latent virus. In the absence of prophylaxis, acute infection typically occurs between the first and third months following transplantation, a period when the transplant recipient's immunosuppression status is at the maximum [8]. The onset of acute infection can however be delayed by the use of prophylactic antiviral agents in the early post-transplant period [8]. However, cytomegalovirus infection can occur later in the first year of transplant after the cessation of antiviral prophylaxis [9] [10]. In a review of the Nigerian experience of kidney transplantation, Arogundade [11] noted that CMV infection and septicaemia accounted for $68.4 \%$ of the mortality in the transplant recipients.

Serologic screening for antibodies to CMV is usually performed on both transplant recipients and their allograft donors before a transplant in order to identify recipients at risk of post-transplant CMV infection who might benefit from preventive strategies [12]. Seronegative recipients of organs from seropositive donors are at the highest risk and in the absence of prophylaxis CMV infection has been reported to occur in 65\% - 88\% recipients while CMV disease may develop in $48 \%$ - $60 \%$ of recipients without CMV prophylaxis [7] [13] [14].

There are two approaches to CMV prevention in transplant recipients namely universal prophylaxis and preemptive therapy. In universal prophylaxis, antiviral therapy is given to all at-risk patients starting at the time of transplant or immediately after transplant for a definite time period [8]. In preemptive therapy, patients are monitored at regular intervals for early evidence of CMV replication prior to the onset of clinical symptoms by use of a laboratory assay [15]. Transplant recipients with early viral replication are treated with antiviral therapy to prevent symptomatic disease [8]. Each approach has its advantages and disadvantages that have to be evaluated in the context of the transplant recipient and the allograft [16]. Preemptive therapy may lower drug costs and reduce toxicity 
but it requires excellent logistic coordination in order to obtain, receive, and act on results in a timely fashion [8]. This can be challenging in low-resource settings like Nigeria where the necessary infrastructure for laboratory testing is lacking coupled with the burden of the cost of care being squarely on the patients in most cases.

Balfour et al. [17] in a randomized, placebo-controlled trial carried out in 104 renal transplant recipients from day 0 of transplant to week 12 found that CMV infection was reduced to $36 \%$ in recipients treated with acyclovir, a synthetic analogue of the purine nucleoside deoxyguanosine compared to $61 \%$ in the placebo group with even greater efficacy on CMV disease (reducing from $29.0 \%$ to $7.5 \%$ ). The dose regimen was $800 \mathrm{mg}$ every 6 hours adjusted to Glomerular Filtration Rate (GFR), with a maximum dose was $3200 \mathrm{mg} /$ day. With pre-transplant $\mathrm{CMV}$ status of $\mathrm{D}^{+} / \mathrm{R}^{-}$combination, post-transplant $\mathrm{CMV}$ infection in the treated group was reduced to $17 \%$ unlike in the placebo group where there was no reduction (17\% versus 100\%). Balfour et al. [17] recommended a prophylactic dose of $3200 \mathrm{mg} /$ day of acyclovir over 12 weeks for renal transplant recipients with normal GFR.

The beneficial effect of another synthetic analogue of deoxyguanosine and CMV prophylactic agent, ganciclovir was also evaluated by Hibbered et al. [18] in a randomized controlled trial. They studied 113 renal transplant recipients who were positive for CMV-IgG antibody. The patients were randomly assigned to receive intravenous ganciclovir ( $2.5 \mathrm{mg} / \mathrm{kg}$ body weight) on every day that Anti-Lymphocyte Globulin (ALG) was administered, or no anti-CMV therapy. The patients were subsequently observed for six months after completion of ALG therapy for the development of CMV disease. The disease occurred in $14 \%$ of recipients who received preemptive ganciclovir therapy compared to $33 \%$ of the control subjects. Furthermore, asymptomatic CMV infection detected by isolation of CMV from buffy-coat specimens occurred in $17 \%$ of the ganciclovir-treated patients and in $35 \%$ of the controls. Preemptive ganciclovir therapy remained protective against CMV disease after controlling for induction or treatment of rejection for which ALG therapy was indicated (adjusted RR-0.27). No adverse events were attributable to ganciclovir therapy during or within six months of its administration. Hibbered et al. concluded that preemptive ganciclovir therapy administered during courses of treatment with ALG reduced the excess occurrence of CMV disease in CMV-IgG positive renal transplant recipients [18].

Also, Couchoud et al. [19] in a meta-analysis of 13 prospective randomized studies evaluated the effectiveness of ganciclovir and acyclovir for cytomegalovirus prophylaxis among recipients of solid organ transplants. Their findings revealed that compared to placebo or no treatment, antiviral therapy resulted in a significant decrease of both CMV disease and infection (relative risk (RR) 0.50 and 0.74 respectively). Both antiviral agents were associated with a decrease in disease, but only ganciclovir lowered the risk of infection. The recommendation for ganciclovir was intravenous treatment for at least 14 days at a dose of $5 \mathrm{mg} / \mathrm{kg}$ twice daily adjusted to GFR. Oral ganciclovir is also available with good bioavai- 
lability. The recommended oral dose was $1 \mathrm{~g}$ three times daily adjusted to GFR and given for a longer period of two to twelve weeks [19].

Hodson et al. [20] in another meta-analysis of controlled trials on prophylaxis with antiviral medications to prevent CMV disease and early death in recipients of solid organ transplants, analyzed 19 trials involving 1981 patients for CMV disease and 17 trials involving 1786 patients for CMV infection. In the study, they found that prophylaxis with acyclovir, ganciclovir or Valacyclovir significantly reduced the risk of CMV disease by $60 \%$ (RR-0.42) and the risk of CMV infections (RR-0.61). Hodson et al. [20] also observed that ganciclovir was more effective than acyclovir in preventing CMV infection (RR-0.45). Furthermore, CMV prophylaxis lowered all-cause mortality by $40 \%$ as a result of reduced mortality from CMV disease [20]. Moreover, the benefits were consistent across recipients of renal, heart and liver transplants. They occurred in both CMV-positive and CMV-negative recipients of organs from CMV-positive donors irrespective of whether immunosuppression included anti-lymphocyte antibody therapy or not. In terms of direct comparisons of the efficacy of prophylactic agents, ganciclovir was more effective than acyclovir in preventing cytomegalovirus disease. Valacyclovir and intravenous ganciclovir were as effective as oral ganciclovir [20]. Hodson et al. recommended that antiviral prophylaxis should be used routinely in both cytomegalovirus positive recipients and cytomegalovirus negative recipients of organs positive for the virus [20].

Valacyclovir is a pro-drug transformed to acyclovir with its bioavailability increased 3 - 5 times. In a randomized, placebo-controlled trial by Lowance et al. [21], the effect was most pronounced in the $\mathrm{D}^{+} / \mathrm{R}^{-}$combination with a reduction of CMV disease at three months from $45 \%$ to $3 \%$ and at six months from $45 \%$ to $16 \%$. In seropositive recipients, the reduction was from $6 \%$ to $0 \%$ at three months and from $6 \%$ to $1 \%$ at six months. In addition, a significant reduction from $52 \%$ to $26 \%$ was shown in acute rejection frequency. The dose administered was $2 \mathrm{~g}$ four times daily over 90 days adjusted to GFR.

In the Nigerian context, acyclovir is more readily available for CMV prophylaxis in transplant units while ganciclovir and its pro-drug valganciclovir are relatively unavailable [11] due to the relatively high cost of the latter two antiviral agents.

\section{Subjects and Methods}

This was a cross-sectional study to evaluate the benefit of prophylaxis with acyclovir on post-transplant CMV as detected by seropositivity for CMV-IgM in the study subjects.

The subjects recruited in the study comprised of renal transplant recipients attending post-transplant follow-up clinics at the Lagos University Teaching Hospital (LUTH) Lagos, St. Nicholas Hospital (SNH) Lagos and Life Support Medical Centre (LSMC) Ikeja, Lagos between October 2004 and July 2005.

The inclusion criteria comprised recipients who had a renal transplant two months or more prior to the study. 
The exclusion criteria were renal transplant recipients with a history of connective tissue disease, and recipients with a history of Varicella zoster infection in the six months preceding the study.

The equation used to calculate minimum sample size in the study was [22]:

$$
n=Z^{2} P q / d^{2}
$$

where:

$n=$ minimum sample size;

$Z=$ normal standard deviation (which corresponds to the desired confidence for the study at a $95 \%$ confidence interval) $[Z=1.96]$;

$P=$ Prevalence;

$q=1-$ Prevalence;

$d=$ Precision set at 0.05 .

The sample size was determined from $80 \%$ prevalence rate in the following equation:

$$
n=\left(1.96^{2} \times 0.8 \times 0.2\right) / 0.05^{2}=246
$$

However, using the equation [22]:

$$
n f=n \div\{1+(n / N)\}
$$

where:

$n f=$ the desired sample size when the entire study population size is less than 10,000 .

$N=$ the estimate of the study population size which was 85 renal transplant recipients in the study area:

$$
n f=246 \div\{1+(246 / 85)\}=63
$$

However, a pilot study of the three centres following up renal transplant recipients in Lagos showed the following number of recipients being followed up at the respective centres during the period of study:

St. Nicholas Hospital, Lagos. $\quad-40$

Lagos University Teaching Hospital, Lagos. $\quad-5$

Life Support Medical Centre, Ikeja, Lagos $\quad-7$

One (1) recipient was reporting to all three centres.

This gave a total of 53 transplant recipients being followed up in the study environment at the time of the study.

Of this number, 40 consented to and participated in the study. The break down from the centres was as followed:

St. Nicholas Hospital.

Lagos University Teaching Hospital. $\quad-4$

Life Support Medical Centre. $\quad-2$

One recipient who participated in the study was reporting to all three centres.

The pre-transplant qualitative CMV-IgG screening results of 27 recipients and their donors as well as data on the use of CMV prophylaxis were obtained from the hospital case records of the study subjects. Data on immunosuppressive drug 
regimens of the study participants were also obtained from the hospital case records of the transplant recipients.

The Microsoft Excel and EPI-Info 2002 statistical software were used for data entry and analysis. Frequency distributions were generated for nominal and ordinal variables while measures of central tendency i.e. mean plus standard deviation were computed for quantitative variables. Variability was expressed as the Standard Deviation (SD). The Chi-square (two-tailed) and Fisher exact tests were employed for comparison of seroprevalence indices for CMV-IgM. Statistically significance was attained with $\mathrm{p}$ value less than 0.05 .

\section{Procedures for Detection of CMV Infection in the Study Subjects}

Blood sample specimen of 4 to $5 \mathrm{ml}$ was obtained from each study subject and control by simple venepuncture at the cubital fossa after observing appropriate aseptic precautions. Each sample was centrifuged to obtain serum specimen, which was stored at $-80^{\circ} \mathrm{C}$ in a deep freezer at the Nigerian Institute of Medical Research (NIMR) Human Virology Laboratory, Lagos until tested.

Samples were collected and pooled between October 2004 and February 2005 for the first batch, and in March 2005 for the second batch. The third batch of specimen samples was collected between April and June 2005 while the fourth batch was collected in July 2005. The serological tests on the samples were done on the four batches on 9th March 2005, 4th April 2005, 29th June 2005, and 25th July 2005 respectively.

The ELISA technique was performed using kits intended for semi-quantitative determination of CMV-IgM (Capita $\left.{ }^{\mathrm{TM}} \mathrm{CMV}-\operatorname{IgM}\right)$ ) antibodies in the test sera. The kits used were from Trinity Biotech Plc (Bray, Ireland) [23]. The technique for CMV ELISA was performed according to the manufacturer's instructions. The CMV-IgM test kit was designed to eliminate errors introduced by the rheumatoid factor, which in the presence of CMV-specific IgG may result in a false positive CMV-IgM reaction. The absorbent solution used in the IgM test kit diminishes competing virus-specific IgG and minimizes rheumatoid factor interference in samples.

\section{CMV-IgM}

For each test sample specimen, the sample absorbance was determined by subtracting the control antigen well absorbance from the antigen well absorbance.

The calibration cut off value (COV) was then determined from the mean optical densities of each pair of calibrator wells and correction factor as contained in the manufacturer's instructions. Immune Status Ratio (ISR) was then calculated as follows [23]:

ISR was then calculated as follows:

Sample Absorbance/COV = ISR;

$$
\begin{aligned}
& \text { ISR }<0.90 \quad=\text { Negative; } \\
& 0.91-1.09=\text { Equivocal; } \\
& >1.10=\text { Positive. }
\end{aligned}
$$


For the CMV-IgM ELISA, the relative sensitivity of the Trinity Biotech kits has been determined as $97.2 \%$ while the relative specificity was determined as $99.2 \%$ [23].

The ELISA tests conducted in this study satisfied all the quality control indices outlined in the manufacturer's instructional manual accompanying each of the test kits. False positive IgM responses to CMV may occur in patients infected with Varicella zoster virus. This advised the exclusion of recipients with a history of Varicella zoster infection in the study. Also, The CMV-IgM test kit was designed to eliminate errors introduced by the rheumatoid factor, which in the presence of CMV-specific IgG may result in a false positive CMV-IgM reaction. The absorbent solution used in the IgM test kit diminishes competing virus-specific IgG and minimizes rheumatoid factor interference in samples.

\section{Results}

The transplant recipients' sociodemographic characteristics are illustrated in Figure 1 and Figure 2. A total of 40 renal transplant recipients were involved in the study. Thirty-two recipients (80\%) were males and eight $(20 \%)$ were females giving a male to female ratio of 4:1 (Figure 1). The mean age of the recipients was $39 \pm 11.6$ years old (range $17-57$ years). In terms of age group distribution of transplant recipients, 2 recipients were less than 20 years old; 18 recipients were in the age group of 21 - 40 years old and 20 recipients were in the age group of 41 - 60 years old (Figure 2).

Cytomegalovirus prophylaxis was received by 16 (40\%) of the recipients. Fifteen of them had oral acyclovir (400 mg 8 hourly) for six months, and one transplant recipient had intravenous ganciclovir for three weeks. Twenty-four recipients $(60 \%)$ received no prophylactic therapy for CMV (Table 1). There was no

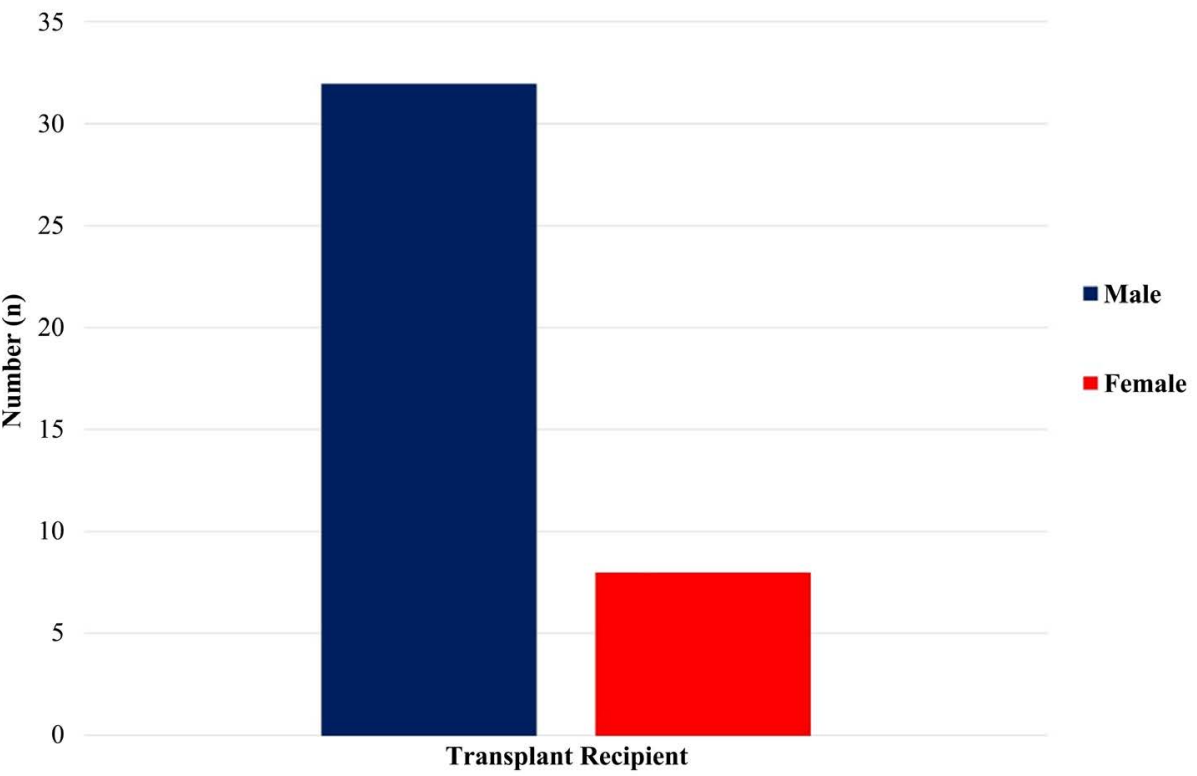

Figure 1. Number and gender distribution of kidney transplant recipients. 


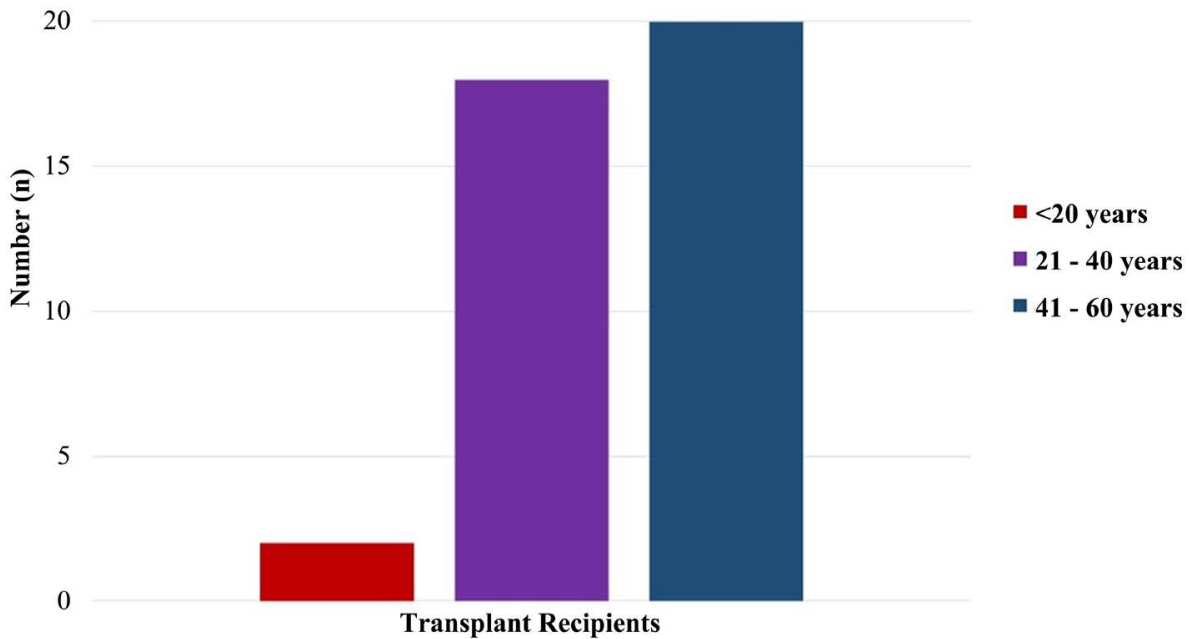

Figure 2. Age distribution of kidney transplant recipients.

significant difference in the prevalence of seropositive CMV-IgM between those who used CMV prophylaxis (oral acyclovir) and the recipients who did not (Fisher exact $\mathrm{p}=0.45)$ (Table 1$)$. One recipient, who received intravenous ganciclovir for three weeks following therapy with basilximab for acute rejection episode in the first week of transplantation, was CMV-IgM negative in the study. Table 1 shows details of the immunosuppressive regimes used by the transplant recipients.

Table 1. Clinical characteristics of renal transplant recipients.

\begin{tabular}{|c|c|c|c|}
\hline \multirow[t]{2}{*}{ Study Population } & \multicolumn{2}{|c|}{ Seropositive CMV-IgM (\%) } & \multirow[t]{2}{*}{ Equivocal } \\
\hline & Positive & Negative & \\
\hline \multirow[t]{3}{*}{ Recipients $(n=40)$} & $9(22.5 \%)$ & $29(72.5 \%)$ & $2(5 \%)$ \\
\hline & \multicolumn{2}{|c|}{ Use of CMV Prophylaxis } & \\
\hline & Acyclovir & Ganciclovir & No Prophylaxis \\
\hline Recipients $(n=40)$ & $15(37.5 \%)$ & $1(2.5 \%)$ & $24(60 \%)$ \\
\hline \multirow{2}{*}{\multicolumn{2}{|c|}{ Immunosuppressive Regimen }} & \multicolumn{2}{|c|}{ Number of Recipients (\%) } \\
\hline & & \multicolumn{2}{|c|}{$n=40$} \\
\hline \multicolumn{2}{|c|}{ CPA } & \multicolumn{2}{|c|}{$14(35 \%)$} \\
\hline \multicolumn{2}{|c|}{ CPM } & \multicolumn{2}{|c|}{$22(55 \%)$} \\
\hline \multicolumn{2}{|c|}{ Others* } & \multicolumn{2}{|c|}{$4(10 \%)$} \\
\hline \multicolumn{4}{|c|}{ Acute rejection episodes } \\
\hline \multicolumn{2}{|c|}{ Yes } & \multicolumn{2}{|c|}{$20(50 \%)$} \\
\hline \multicolumn{2}{|c|}{ No } & \multicolumn{2}{|c|}{$20(50 \%)$} \\
\hline
\end{tabular}

CMV prophylaxis (oral acyclovir $400 \mathrm{mg} 8$ hourly for six months) versus No prophylaxis (Fisher exact $\mathrm{p}=$ 0.45). CPA-Cyclosporine/Prednisolone/Azathioprine; CPM-Cyclosporine/Prednisolone/Mycophenolate mofetil; ${ }^{*}$ Prednisolone/Mycophenolate mofetil/Sirolimus-2; Prednisolone/Mycophenolate/Tacrolimus-1; Cyclosporine/Prednisolone-1. 
The post-transplant duration in the recipients studied ranged from 2 to 80 months (mean: $17.6 \pm 18.6$ months). Thirty-one recipients (77.5\%) had been transplanted for more than four months before the study. Nine (22.5\%) had their transplants two to four months before the study. Thus, the transplant recipients had been followed up for an average of $17-18$ months by the time of this study.

The pre-transplant qualitative CMV-IgG screening test records were available for 27 of the recipients and their allograft donors. In the 27 recipients, 26 (96.3\%) were seropositive, and one (3.7\%) was seronegative. For the allograft donors, 25 of the 27 (92.6\%) were seropositive for CMV IgG while two (7.4\%) were seronegative. The renal allograft donor to the sole pre-transplant CMV-IgG seronegative recipient in the study was also seronegative for CMV-IgG prior to the transplant.

\section{Discussion}

This was a pioneering study on benefit of CMV prophylaxis in renal transplant recipients in Nigeria. The study was carried out about 16 years ago as part of a Nephrology fellowship dissertation on the Prevalence of Cytomegalovirus infection among renal transplant recipients and their donors in Lagos, Nigeria [24]. Since the first kidney transplant was carried out at Saint Nicholas Hospital, Lagos in March 2000, several kidney transplant units have been established across different geographical regions in the country and more than 200 kidney transplants have now been carried out by the transplant centres [2]. Despite the growing number of transplant centres and successful kidney transplants in Nigeria, no studies relating to the subject of this paper have been published in literature.

This study found CMV prophylaxis with acyclovir for six months showed no significant benefit on post-transplant CMV infection prevention in renal transplant recipients. Those who received the prophylaxis did not differ from the untreated group with respect to CMV-IgM seroprevalence. The finding differs from that of Balfour et al. who observed significant reduction in both CMV infection and disease in a study of 104 transplant recipients treated with a twelve-week course of acyclovir ( $800 \mathrm{mg} 6$ hourly). However, the dosage of acyclovir taken by recipients in this study ( $400 \mathrm{mg} 8$ hourly for six months) was below the regime of $800 \mathrm{mg} 6$ hourly for three months employed by Balfour et al. [17] although the prophylactic therapy in this study was for a longer duration. Again, contrary to the finding in this study, Hodson et al. [20] in a meta-analysis of controlled trials on prophylaxis with antiviral medications to prevent CMV disease and early death in recipients of solid organ transplants also found that prophylaxis with acyclovir significantly reduced the risk of CMV disease (RR-0.42) and the risk of CMV infection (RR-0.61). However, ganciclovir was more effective than acyclovir in preventing CMV infection [20].

On the other hand, Couchoud et al. [19] in a meta-analysis of 13 prospective randomized studies found that although prophylaxis with acyclovir was associated with significant reduction in post-transplant CMV disease in transplant recipients however, like the observation in this study it was not associated with 
significant reduction in CMV infection. In this study none of the transplant recipients had CMV disease at the time of study but this observation might be related to the fact that the mean post-transplant duration at which the recipients were tested for CMV infection in this study was long (17.6 \pm 18.6 months). In renal transplantation, the risk of CMV disease is highest in the second month following transplantation and is largely determined by the serologic status of the donor and recipient for the virus [13] [25]. The pre-transplant donor/recipient CMV serologic status combination in the majority of cases in this study was $\mathrm{D}^{+} / \mathrm{R}^{+}$. Only one transplant recipient in this study had a seronegative pre-transplant CMV-IgG status and a CMV-IgG seronegative donor ( $\mathrm{D}^{-} / \mathrm{R}^{-}$combination). This recipient was also seronegative for post-transplant CMV infection.

Another transplant recipient in this study was treated with intravenous ganciclovir as preemptive therapy following treatment with basilximab for acute rejection episode. The recipient was seronegative for CMV-IgM during the study. This beneficial effect of preemptive ganciclovir therapy has also been reported in several other studies. For instance, Hibbered et al. [18] evaluated the beneficial effect of ganciclovir in a randomized controlled trial involving 113 renal transplant recipients who were positive for CMV-IgG antibody and who were randomly assigned to receive intravenous ganciclovir $(2.5 \mathrm{mg} / \mathrm{kg}$ body weight) on every day that anti-lymphocyte globulin (ALG) was administered, or no anti-CMV therapy. The patients were observed for six months after completion of ALG therapy for development of CMV disease. They found that preemptive ganciclovir therapy administered during courses of treatment with ALG reduced the excess occurrence of CMV disease in CMV-IgG positive renal transplant recipients [18]. Furthermore, asymptomatic CMV infection as detected by isolation of CMV from buffy-coat specimens occurred in 17\% of the ganciclovir treated patients and $35 \%$ of the control subjects. Moreover, preemptive ganciclovir therapy remained protective against CMV disease after controlling for induction or treatment of rejection for which ALG therapy was indicated (adjusted RR-0.27) and there were no adverse events attributable to ganciclovir therapy during or within six months of its administration.

Other studies have noted greater benefit with ganciclovir in comparison to acyclovir. Couchoud et al. [19] in a meta-analysis of 13 prospective randomized studies evaluated the effectiveness of ganciclovir and acyclovir for cytomegalovirus prophylaxis among recipients of solid organ transplants and observed that while both antiviral agents were associated with a decrease in CMV disease, only ganciclovir lowered the risk of infection.

The recommendation for ganciclovir was intravenous treatment for at least 14 days at a dose of $5 \mathrm{mg} / \mathrm{kg}$ twice daily adjusted to GFR. Oral ganciclovir is also available with good bioavailability at a recommended dose of $1 \mathrm{~g}$ three times daily adjusted to GFR and given for a period of two to twelve weeks and is as effective as intravenous ganciclovir [19] [20]. Hodson et al. [20] in another meta-analysis of controlled trials also observed that ganciclovir was more effective than acyclovir in preventing CMV infection and disease. They recommended that 
antiviral prophylaxis should be used routinely in both cytomegalovirus positive recipients and cytomegalovirus negative recipients of organs positive for the virus [20].

In this study, only $40 \%$ of transplant recipients had any form of antiviral prophylaxis despite the pre-transplant CMV serologic status of $\mathrm{D}^{+} \mathrm{R}^{+}$combination in most of the transplant recipients whose pre-transplant CMV serology test results were available. The lack of access to antiviral prophylaxis for most of the transplant recipients in this study is related to the huge financial burden the transplant procedure and post-transplant care imposes on the recipients who in most cases have to bear the costs by themselves [11]. The average cost of a sixmonth oral course of acyclovir at the prescribed dose of $400 \mathrm{mg} 8$ hourly taken by recipients in this study is in the region of $\$ 305$ (USD) which is equivalent to 126,000 Naira (Nigeria local currency). This amount represents 70 per cent of the country's minimum wage for the same period of six mouths.

\section{Conclusion}

Routine antiviral prophylaxis is an effective preventive measure against post-transplant CMV infection and disease in transplant recipients. Studies have shown a greater benefit with ganciclovir compared to acyclovir in the prevention of post-transplant CMV infection and disease. The only transplant recipient in this study who had preemptive intravenous ganciclovir therapy did not have post-transplant CMV infection. The lack of significant benefit of acyclovir prophylaxis in preventing posttransplant CMV infection observed in this study aligns with other studies that have shown the superiority of ganciclovir over acyclovir. However, the relatively higher cost of ganciclovir has limited its availability to renal transplant recipients in the study environment.

\section{Strengths and Limitations}

This simple study was carried out between 2004 and 2005 at a time when kidney transplantation in Nigeria was still in its infancy. It is the first and till date the only study to evaluate the benefit of antiviral prophylaxis on post-transplant cytomegalovirus infection prevention in kidney transplant recipients in the study environment.

The number of transplant recipients in this study was |relatively small compared with those in other parts of the world where kidney transplantation has been established for several decades.

\section{Acknowledgements}

This author is grateful to all the participants in this study for their willingness to participate. The author is also grateful to three post-renal transplants follow-up centres from which the study participants were recruited.

\section{Data Availability}

All the necessary data are included in the manuscript. 


\section{Ethics Declaration}

The Research and Ethics Committee of Lagos University Teaching Hospital Lagos, the Management of Saint Nicholas Hospital Lagos, and the Management of Life Support Medical Centre Ikeja approved the study.

\section{Informed Consent}

Informed written consent was obtained from all the study participants.

\section{Consent for Publication}

The author gives his consent for this study to be published.

\section{Conflicts of Interest}

The author declares no conflicts of interest regarding the publication of this paper.

\section{References}

[1] Goodman, W.G. and Danovitch G.M. (2001) Options for Patients with End-Stage Renal Disease. In: Danovitch, G., Ed., Handbook of Kidney Transplantation, 3rd Edition, Lippincott Williams and Wilkins, Philadelphia, 1-16.

[2] Okafor, U.H. (2016) Kidney Transplant in Nigeria: A Single Centre Experience. Pan African Medical Journal, 25, Article No. 112.

https://doi.org/10.11604/pamj.2016.25.112.7930

[3] Fishman, J.A. and Rubin, R.H. (1998) Infection in Organ Transplant Recipients. New England Journal of Medicine, 338, 1741-1751. https://doi.org/10.1056/NEJM199806113382407

[4] Rubin, R.H. (1993) Infectious Disease Complications of Renal Transplantation. Kidney International, 44, 221-236. https://doi.org/10.1038/ki.1993.234

[5] Liapis, H., Storch, G.A., Ashley Hill, D., Rueda, J. and Brennan, D.C. (2003) CMV Infection of the Renal Allograft Is Much More Common Than the Pathology Indicates: A Retrospective Analysis of Qualitative and Quantitative Buffy Coat CMV-PCR, Renal Biopsy Pathology and Tissue CMV-PCR. Nephrology Dialysis Transplantation, 18, 397-402. https://doi.org/10.1093/ndt/18.2.397

[6] Patel, R. and Paya, C.V. (1997) Infections in Solid-Organ Transplant Recipients. Clinical Microbiology Reviews, 10, 86-124. https://doi.org/10.1128/CMR.10.1.86

[7] Hartmann, A., Sagedal, S. and Hjelmesæth, J. (2006) The Natural Course of Cytomegalovirus Infection and Disease in Renal Transplant Recipients. Transplantation, 82, S15-S17. https://doi.org/10.1097/01.tp.0000230460.42558.b0

[8] Cukuranovic, J., Ugrenovic, S., Jovanovic, I., Visnjic, M. and Stefanovic, V. (2012) Viral Infection in Renal Transplant Recipients. The Scientific World Journal, 2012, Article ID 820621. https://doi.org/10.1100/2012/820621

[9] Weikert, B.C. and Blumberg, E.A. (2008) Viral Infection after Renal Transplantation: Surveillance and Management. Clinical Journal of the American Society of Nephrology, 3, S76-S86. https://doi.org/10.2215/CJN.02900707

[10] Green, M., Avery, R. and Preiksaitis, J. (2004) Guidelines for the Prevention and Management of Infectious Complications of Solid Organ Transplantation. American Journal of Transplantation, 4, 160-163.

https://doi.org/10.1111/j.1600-6135.2004.00737.x 
[11] Arogundade, F.A. (2013) Kidney Transplantation in a Low-Resource Setting: Nigeria Experience. Kidney International, 3, 241-245.

https://doi.org/10.1038/kisup.2013.23

[12] Fischer, S.A and Avery, R.K. (2009) Screening of Donor and Recipient prior to Solid Organ Transplantation. American Journal of Transplantation, 9, S7-S18. https://doi.org/10.1111/j.1600-6143.2009.02888.x

[13] Brennan, D.C. (2001) Cytomegalovirus in Renal Transplantation. Journal of American Society of Nephrology, 12, 848-855. https://doi.org/10.1681/ASN.V124848

[14] Mwintshi, K. and Brennan, D.C. (2007) Prevention and Management of Cytomegalovirus Infection in Solid-organ Transplantation. Expert Review of Anti-Infective Therapy, 5, 295-304. https://doi.org/10.1586/14787210.5.2.295

[15] Rubin, R.H. (2005) Transplant Infectious Disease: A Work in Progress. Transplant Infectious Disease, 7, 49-50. https://doi.org/10.1111/j.1399-3062.2005.00101.x

[16] Paya, C.V. (2001) Prevention of Cytomegalovirus Disease in Recipients of Solid Organ Transplants. Clinical Infectious Diseases, 32, 596-603. https://doi.org/10.1086/318724

[17] Balfour, H.H., Chace B.A., Stapleton, J.T., Simmons, R.L. and Fryd, D.S. (1989) A Randomized Placebo-Controlled Trial of Oral Acyclovir for the Prevention of Cytomegaloviruus Disease in Recipients of Renal Allografts. New England Journal of Medicine, 320, 1381-1387. https://doi.org/10.1056/NEJM198905253202105

[18] Hibberd, P.L., Tolkoff-Rubin, N.E., Conti, D., et al. (1995) Preemptive Ganciclovir Therapy to Prevent Cytomegalovirus Disease in Cytomegavirus Antibody Positive Renal Transplant Recipients. A Randomized Controlled Trial. Annals of Internal Medicine, 123, 18-26. https://doi.org/10.7326/0003-4819-123-1-199507010-00002

[19] Couchoud, C., Cucherat, M., Haugh, M. and Pounteil-Noble, C. (1997) Cytomegalovirus Prophylaxis with Antiviral Agents in Solid Organ Transplantation: A Meta-Analysis of Recipients. Transplantation, 64, 1843-1846

[20] Hodson, E.M., Jones, C.A., Webster, A.C., Stripped, G.M., Barday, P.G., Kable, K., et al. (2005) Antiviral Medications to Prevent Cytomegalovirus Disease and Early Death in Recipients of Solid-Organ Transplants: A Systematic Review of Randomized Controlled Trials. Lancet, 365, 2105-2115. https://doi.org/10.1016/S0140-6736(05)66553-1

[21] Lowance, D., Neumayer, H.H, Legender, C.M., et al. (1999) Valacyclovir for the Prevention of Cytomegalovirus Disease after Renal Transplantation. International Valacyclovir Cytomegalovirus Prophylaxis Transplantation Study Group. The New England Journal of Medicine, 1340, 1462-1470. https://doi.org/10.1056/NEJM199905133401903

[22] Araoye, M.O. (2004) Subjects Selection. In: Araoye, M.O., Ed., Research Methodology with Statistics for Health and Social Sciences, Nathadex Publishers, Ilorin, 115-129.

[23] Trinity Biotech Capita (2001) Cytomegalovirus IgG and IgM ELISA Test Instructional Manual. Trinity Biotech PLC., Bray, Ireland.

[24] Menakaya, N.C. (2006) Prevalence of Cytomegalovirus Infection among Renal Transplant Recipients and Their Donors in Lagos, Nigeria. Nephrology Fellowship Dissertation, National Postgraduate Medical College of Nigeria, National Postgraduate Medical College of Nigeria, Unpublished.

[25] Hirata, M., Terasaki, P.I. and Cho, Y.W (1996) Cytomegalovirus Antibody Status and Renal Transplantation: 1987-1994. Transplantation, 62, 34-37.

https://doi.org/10.1097/00007890-199607150-00007 